\title{
Teacher Location Tracking System in College Campus
}

\author{
Sayali A. Kumbhar \\ UG students, Dept. Of Information \\ Technology Vidyalankar Institute \\ Of Technology,Wadala Road \\ Mumbai University, Mumbai, India \\ Aditya Birla Hostel \\ Chembur,Mumbai
}

\author{
Roshni N. Gore \\ UG students, Dept. Of Information \\ Technology Vidyalankar Institute \\ Of Technology, Wadala Road \\ Mumbai University, Mumbai, India \\ Tulsi plaza near abhinav school \\ Asangaon Mumbai
}

\author{
Vaishali R. Jadhav \\ UG students, Dept. Of Information \\ Technology Vidyalankar Institute \\ Of Technology,Wadala Road \\ Mumbai University, Mumbai, India \\ Gul Mohd Chawl \\ Dharavi Mumbai
}

\begin{abstract}
This Paper shows how to track a teacher's location in the college by using computer based system. This system is very useful to all, especially for huge organizations for finding location of particular person in huge organization. This project intends to introduce more user friendliness in the various activities such as location updates, maintenance, and searching. The searching of a teacher has been made it quite simple as all the details of a teacher can be obtained by simply keying in the identification of that teacher. The entire information has been maintained in the database or Files and whoever teacher wants to retrieve can easily retrieve, only after authorization. Teacher can retrieve the necessary information which can be easily accessible from the file. This system will avoid physical stress and reduce time for finding a teacher in large organization.
\end{abstract}

\section{General Terms}

Your general terms must be any term which can be used for general classification of the submitted material such as Pattern Recognition, Security, Algorithms et. Al.

\section{Keywords}

RFID Tag(Radio-frequency identification),IRSensor(Infrared Radiation),Microcontroller(AT89S52),Zigbee.

\section{INTRODUCTION}

Today, in the organization or in institute when one teacher wants to search another teacher for some work then they can use their mobile phones. By talking on the mobile phone, we get to know where a particular teacher is, but if mobile phones are not reachable, if the phones battery is down, the organization have the phone jammers or some of them don't allow the cell phones at all then there is no easy way to reach out to a person. The second way is, in which a teacher can go in entire organization to search for their colleagues physically. This way is helpful if organization is small but if organization is large then this way is not suitable. Because searching for a particular teacher in the entire organization is a tedious task. So to overcome this problem we have decided to design a system which can easily track a teacher's location in a large organization.

Teacher location tracking system is fully computer based system. Teacher location tracking system involves hardware and software combination. This system is very useful to all, especially for large organizations. This is to enhance and upgrade the existing system by increasing its efficiency and effectiveness. The system improves the working methods by replacing the existing manual system with the computer-based system.
This system will be designed by using microcontroller, IR sensor, RFID transmitter and RFID receiver and zigbee. In this system we are designing two devices. One is carried by a teachers and one device is set in the classroom, labs, canteens, and department or anywhere in college campus. In this project we are using HTML.CSS,PHP for design a server and Diptrace for PCB(Printed circuit Board) layout.

\section{LITERATURE REVIEW}

- MurizahKassim, HasbullahMazlan, NorlizaZaini in their paper "Web-based student attendance system using RFID technology" emphasize on the fact that development of a student attendance system based on Radio Frequency Identification (RFID) technology. The existing conventional requires to physically searching the teacher or phone call the teacher. As common as it seems, such system lacks of automation, where a number of problems may arise. This includes the time unnecessarily consumed by the teachers to find. Having a system that can automatically capture Teachers Location by flashing the Microcontroller and RFID Tag from both transmitter and Receiver side can really save all the mentioned troubles. This is the main motive of our system and in addition having an online system accessible anywhere and anytime can greatly help the lecturers to keep track of their Location in Emergency.

- Lasse Toivanen in their paper RFID-Based Book Finder Locating items rapidly and accurately has become a crucial part of our modern society and industry. Accuratelocating not only saves time and money but also reduces waste, as products do not get lost along supply chains. One promising indoor tracking method is provided by radio-frequency identification (RFID) technology. The main benefit of RFID technology is the ability to inventory items simultaneously and rapidly without the requirement of line of sight to the target.

- In IEEE paper of "RFID based Prepaid Car Parking System" has application in many areas like Industries, Companies, Offices, Shopping malls. We have provided a RFID Tag which is connected to Microcontroller from both Transmitter and Receiver side. Every Time the data of the Teacher will be updated and we can easily find the Location of Teacher.

- Sanghyun Son in their paper "asset tracking system using IEEE 802.15.4a"talk about The real-time location tracking of various vehicle assets is a major factor for efficient port terminal processing. In our system the realtime location tracking assets is a major factor for efficient port terminal processing. In this paper, we will describe a system that we designed for asset tracking based on wireless communications for the efficient 
management of Location Tracking in the Campus. Improved the tracking estimation accuracy between Teachers by using the IR Sensor, RFID tag, Microcontroller etc communication between the light tower's readers and crane's mobile readers.

\section{PROPOSED SYSTEM}

The proposed system helps to find teacher in very short time. Usually, the problem is finding or searching another teacher in a large organization is a tedious task because one teacher need to search that particular person in large organization and also sometimes teacher cannot call to another teacher for searching them because of mobile network unavailability or mobile battery issue. This problem can overcome with help of teacher location tracking system. Teacher location tracking system is designed as computer based system with hardware and software combination. This system always keeps tracking location of teacher and maintains record on the server, so by using this system one teacher can easily track location of another teacher.

\subsection{Process Model Used For The Project}

\subsubsection{Transmitter side}

RFID Tx

Fig. 1 :Transmitter side

Fig 1 is transmitter side device (RFID tag). This device carried by teacher. This device sends its ID to receiver device.

\subsubsection{Receiver Side}

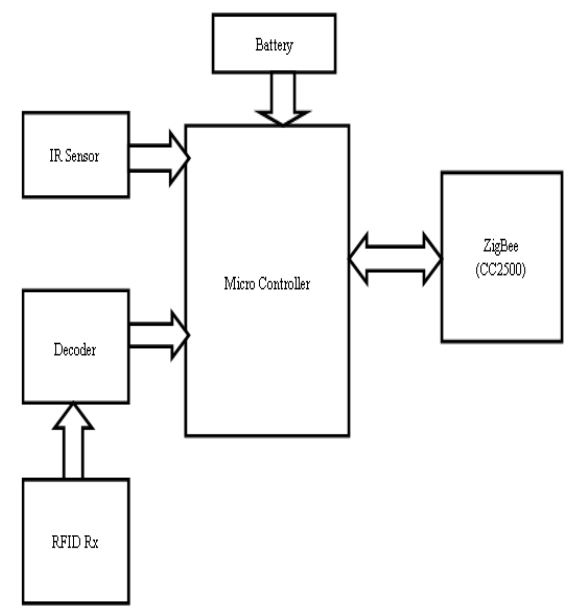

Fig.2: Receiver side
Fig. 2 contain receiver side device which is set in classroom, labs, canteens etc. Which contain IR sensor, battery, RFID receiver, micro controller, zigbee. This device receiver teacher Id and then sends location of that teacher along with teacher ID by using zigbee.
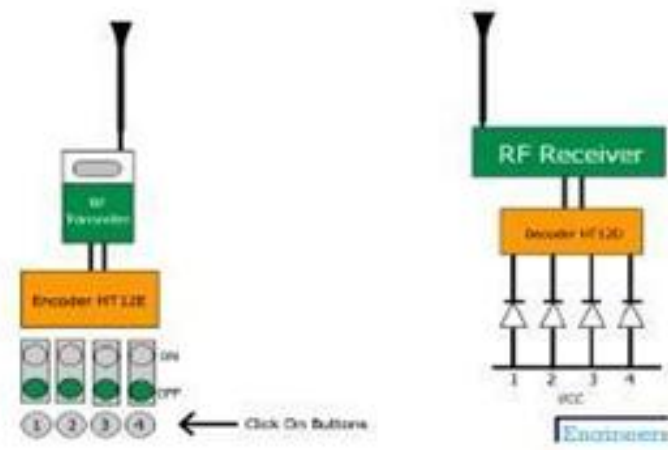

Fig.3: RFID circuit

Fig 1 and fig 2 uses RFID transmitter and receiver respectively .This devices utilizes the RF module (Tx/Rx) for making a wireless remote, which could be used to drive an output from a distant place.

$\mathrm{RF}$ module, as the name suggests, uses radio frequency to send signals. These signals are transmitted at a particular frequency and a baud rate. A receiver can receive these signals only if it is configured for that frequency. This radio frequency (RF) transmission system employs Amplitude Shift Keying (ASK) with transmitter/receiver (Tx/Rx) pair operating at $434 \mathrm{MHz}$. The transmitter module takes serial input and transmits these signals through RF. The transmitted signals are received by the receiver. Module placed away from the source of transmission.

The system allows one way communication between two nodes, namely, transmission and reception. The RF module has been used in conjunction with a set of four channel encoder/decoder ICs. Here HT12E \& HT12D have been used as encoder and decoder respectively. The encoder converts the parallel inputs (from the remote switches) into serial set of signals. These signals are serially transferred through RF to the reception point. The decoder is used after the RF receiver to decode the serial format and retrieve the original signals as outputs. These outputs can be observed on corresponding LEDs.

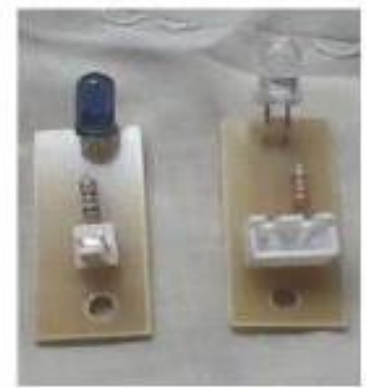

Fig 4: IR sensor

Fig 4 indicate IR sensor. An infrared sensor is an electronic device that emits and/or detects infrared radiation in order to 
sense some aspect of its surroundings. Infrared sensors can measure the heat of an object, as well as detect motion.

IR Sensors work by using a specific light sensor to detect a select light wavelength in the Infra-Red (IR) spectrum. By using an LED which produces light at the same wavelength as what the sensor is looking for, you can look at the intensity of the received light. When an object is close to the sensor, the light rom the LED bounces off the object and into the light sensor. This results in a large jump in the intensity, which we already know can be detected using a threshold.

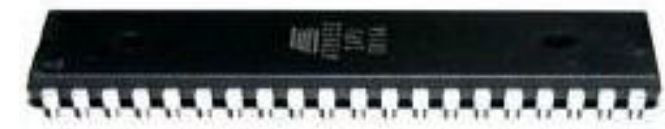

\section{Fig 5 :Micro controller AT89S52}

Fig 5 consist micro controller AT89S52 is a low-power, highperformance CMOS 8-bit microcontroller with $8 \mathrm{~K}$ bytes of insystem programmable Flash memory. The device is manufactured using Atmel's high-density nonvolatile memory technology and is compatible with the industry-standard $80 \mathrm{C} 51$ instruction set and pin out. The on-chip Flash allows the program memory to be reprogrammed in-system or by a conventional nonvolatile memory programmer. By combining a versatile 8-bit CPU with in-system programmable Flash on a monolithic chip, the Atmel AT89S52 is a powerful microcontroller which provides a highly-flexible and costeffective solution to many embedded control applications.

The AT89S52 provides the following standard features: $8 \mathrm{~K}$ bytes of Flash, 256 bytes of RAM, 32 I/O lines, Watchdog timer, two data pointers, three 16-bit timer/counters, a six vector two-level interrupt architecture, a full duplex serial port, on-chip oscillator, and clock circuitry. In addition, the AT89S52 is designed with static logic for operation down to zero frequency and supports two software selectable power saving modes. The Idle Mode stops the CPU while allowing the RAM, timer/counters, serial port, and interrupt system to continue functioning. The Power-down mode saves the RAM con-tents but freezes the oscillator, disabling all other chip functions until the next interrupt or hardware reset.

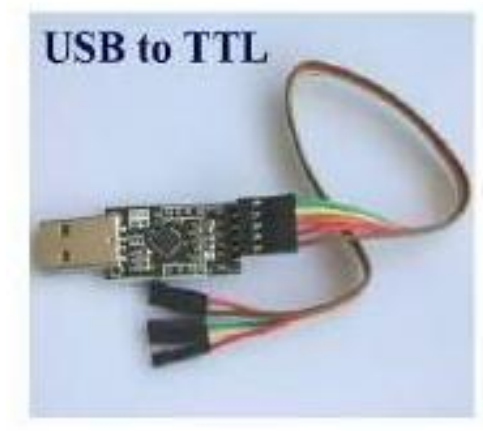

Fig 6 :USB to TTL

In fig 6, the cable is easiest way ever to connect to your microcontroller/Raspberry $\mathrm{Pi} / \mathrm{Wi}-\mathrm{Fi}$ router serial console port.

Inside the big USB plug is a USB <->Serial conversion chip and at the end of the 36 " cable are four wire - red power, black ground, white RX into USB port, and green TX out of the USB port. The power pin provides the $5 \mathrm{~V}, 500 \mathrm{~mA}$ direct from the USB port and the RX/TX pins are $3.3 \mathrm{~V}$ level for interfacing with the most common 3.3V logic level chipsets.

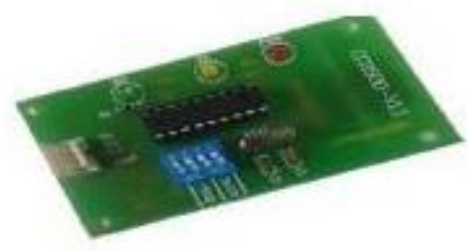

Fig 7 :Zigbee (CC2500) circuit

Fig 7 consist Zigbee CC2500 circuit which is used for data transmission. Zigbee is used at receiver side and at PC side. This circuit is a FSK /MSK Transceiver module. It provide extensive hardware support for packet handling, data buffering, burst transmissions, clear channel assessment, link quality indication and wake on radio. It's data stream can be Manchester coded by the modulator and decoded by the demodulator .It has a high performance and easily to design your product. It can be used in $2400-2483.5 \mathrm{MHz}$ ISM/SRD band systems, Consumer Electronics, Wireless game controllers, Wireless audio wireless Keyboard/Mouse and others wireless system

\subsubsection{Server Side}

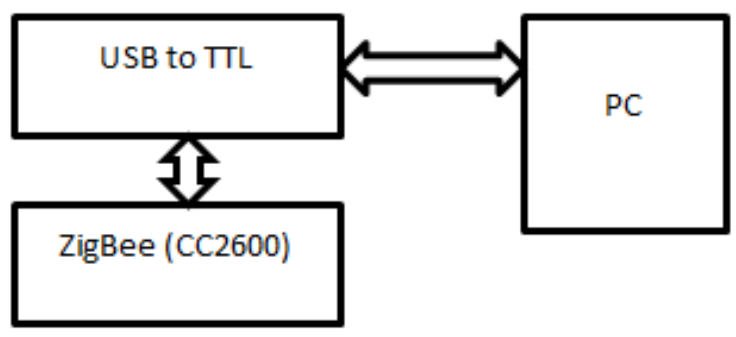

Fig 8: Server side device

Fig 8 consist zigbee(CC2600),USB to TTL which is connected to computer .Server is designed by using programming language PHP, CSS, HTML. When one teacher want to find location of another teacher then teacher need to open this portal and just write particular teacher ID in search box then a location of that particular teacher, display on screen.

\subsection{Methodology}

The problem we are attempting to solve is to develop a system called teacher location tracking system to eliminate problem of searching or finding a teacher in the large organization. Flow of the project is given below: 


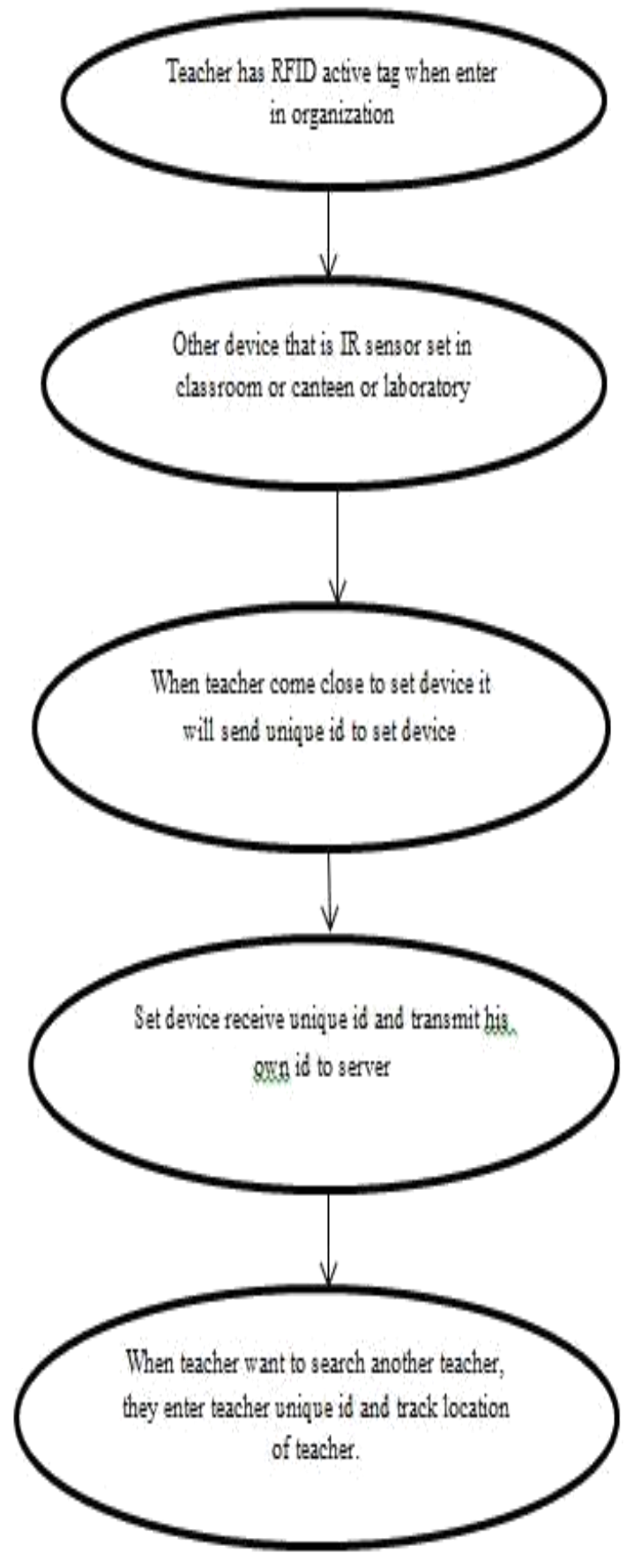

\section{CONCLUSION}

The aim of this system is tracking a teacher's location in the college by using computer based system. The main aim of this system is reducing time for finding a teacher in the large organization and also reduces physical stress.

\section{REFERENCES}

[1] Getting started with rfid, maker media inc.,2012 "In Texas, 28,000 Students Test an Electronic Eye". Nytimes.com. Retrieved 2013-10-17.

[2] "RFID Used for Student Security and Attendance Reporting". Spring ISD. 2010 - 10 - 11 . Retrieved 2013 $-10-12$.

[3] Schofield, Jack (2005 -02-10). "School RFID Plan Gets an F | Technology". Theguardian.com. Retrieved 2013 $10-12$.

[4] http://ieeexplore.ieee.org/document/7454189.

[5] http://ieeexplore.ieee.org/document/74843 